\title{
Pediculotomy as an adjunct to posterior cervical hemilaminectomy, foraminotomy, and discectomy
}

\author{
K. Michael Webb, M.D., George Kaptain, M.D., Jonas Sheehan, M.D., AND \\ John A. Jane, SR., M.D., Ph.D. \\ Department of Neurological Surgery, University of Virginia Health Sciences Center, Charlottesville, \\ Virginia
}

\begin{abstract}
Currently the posterior approach undertaken to perform cervical hemilaminectomy and foraminotomy provides sufficient exposure to treat the majority of lateral soft-disc herniations or osteophytes causing radiculopathy. Limitations imposed by the surgical field, however, often necessitate excessive retraction of the nerve root and epidural venous plexus, which may potentially exacerbate a preexisting radiculopathy or increase intraoperative blood loss. Partial resection of the inferior pedicle augments exposure and enlarges the neural foramen, thus facilitating decompression while minimizing manipulation of the nerve root and epidural venous plexus.

With the patient in the prone position, partial hemilaminectomy and foraminotomy are performed using a highspeed 3-mm diamond burr with continuous irrigation. The thecal sac and nerve root are exposed, and the overlying fibroareolar layer is coagulated and incised. With the nerve root protected and under direct vision, the superomedial portion of the inferior pedicle is removed. Nerve root decompression is then performed through this augmented exposure.

Partial excision of the pedicle allows for more expeditious removal of the pathological elements causing cervical radiculopathy and requires minimal manipulation of the nerve root and epidural venous plexus. This procedure results in a potential decrease in transient postoperative radiculopathy and minimization of intraoperative blood loss. In addition, the resulting foraminal enlargement enhances the decompression provided by traditional foraminotomy, even if discectomy is not performed.
\end{abstract}

\section{KEY WordS • cervical spine • operative technique • pedicle • foraminotomy • cervical radiculopathy}

Combined posterior cervical hemilaminectomy, discectomy, and foraminotomy is a well-described and effective procedure for the treatment of lateral intervertebral disc herniation or spondylosis causing symptomatic nerve root compression. ${ }^{1,2,4,6-8}$ The exposure provides excellent visualization of the nerve root, thecal sac, and epidural venous plexus and allows the surgeon to avoid damaging the major anterior visceral structures and carotid artery. Additionally, the risk of developing adjacent-level disease may be minimized when using a limited posterior approach without subsequent fusion..$^{3-7}$

Although the posterior approach clearly exposes the nerve root and thecal sac, the compressive disease is anterior to the nerve root. In most cases, the exposure provided by the standard approach is adequate to excise the offending disc fragment or osteophyte without disruption of the anterior epidural venous plexus or without requiring undue retraction on the nerve root.

In a significant number of cases, however, complete re- section of soft-disc fragments and osteophytes cannot be performed without excessive retraction on the nerve root and venous plexus, which may temporarily exacerbate the preexisting radiculopathy or increase intraoperative blood loss, obscuring the surgical field. When this occurs, partial removal of the superomedial portion of the inferior pedicle allows for easier and more complete excision of the compressive lesion and, additionally, enlarges the neural foramen and provides a more complete decompression in cases in which discectomy is not performed.

\section{INDICATIONS FOR ADJUNCTIVE PEDICULOTOMY}

At our institution, the posterior cervical approach is favored in patients in whom only neck and arm pain are present and in whom physical examination suggests a stenotic neural foramen, which is caused by a herniated nucleus pulposus or uncovertebral joint spondylosis and 
confirmed by magnetic resonance imaging or computerized tomographic myelography. Patients who experience bilateral arm pain without myelopathy may undergo bilateral hemilaminectomies and foraminotomies. Those with myelopathy or those with a large central disc herniation or osteophyte causing either significant spinal cord compression and/or cord signal change demonstrated on $\mathrm{T}_{2}$ weighted magnetic resonance imaging are considered for anterior discectomy and fusion. Intraoperatively, pediculotomy is performed in cases in which the neural foraminal volume is limited or in which excessive nerve root retraction is necessary to achieve adequate removal of the offending lesion.

\section{SURGICAL TECHNIQUE}

After induction of general anesthesia, the patient is placed in the prone position and the head is immobilized slightly flexed with a pinion headrest. The incision is cen-

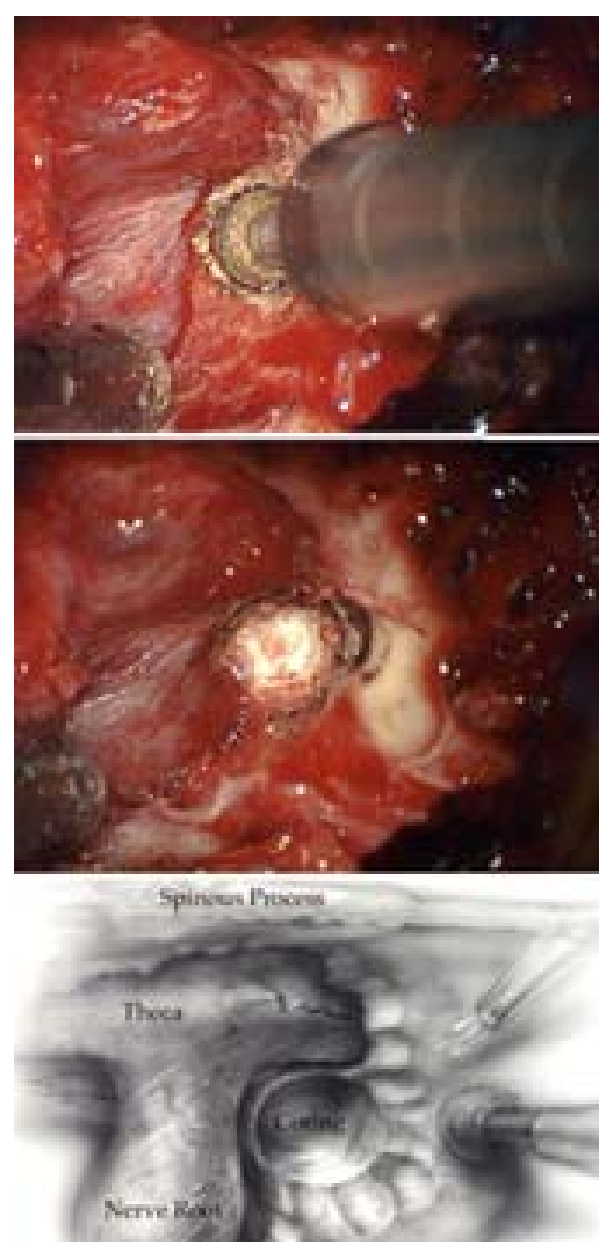

Fig. 1. Upper: Intraoperative photograph demonstrating superomedial pediculotomy of the inferior pedicle performed with a 3-mm diamond bit. Note the thecal sac and exiting nerve root at the bottom of the picture. The fibroareolar layer is intact and overlying the nerve root. Center and Lower: Intraoperative photograph (center) and illustration (lower) demonstrating completed pediculotomy in which the axilla of the exiting nerve root was exposed.

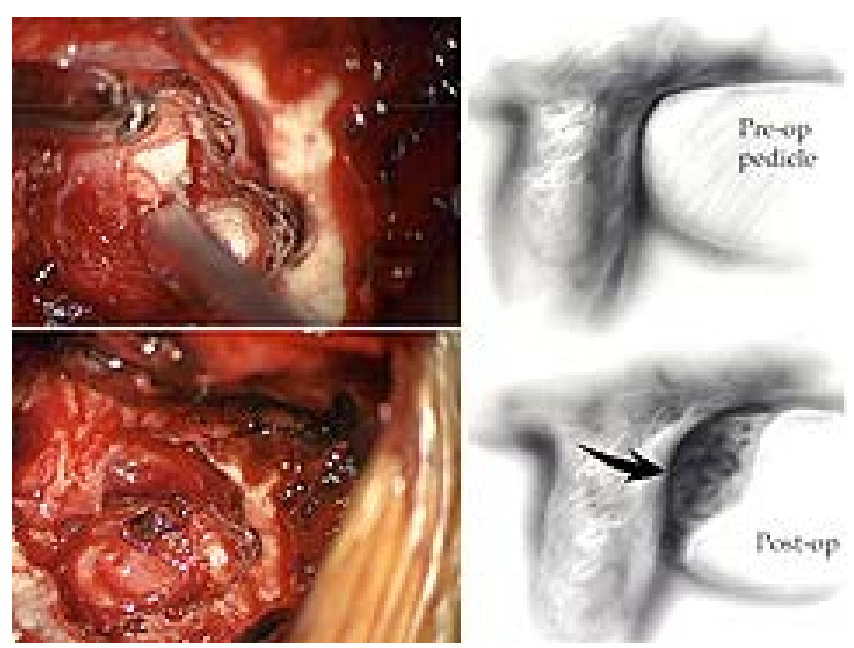

Fig. 2. Upper Left: Intraoperative photograph demonstrating resection of the herniated disc fragments in nerve root axilla via superomedial foraminotomy. Note the improved exposure obtained by superior pediculotomy. Lower Left and Right: Intraoperative photograph (lower right) and illustration (right) showing the completed foraminotomy and discectomy with pediculotomy.

tered over the affected vertebral levels. The subcutaneous tissue, ligamentum nuchae, and cervical musculature are divided in the relatively avascular midline by using sharp dissection or monopolar electrocautery. Two adjacent spinous processes are marked, and a localizing radiograph is obtained. After confirmation of the correct level, unilateral subperiosteal dissection is performed to expose the lamina and facet joint. Using the operating microscope and a high-speed 3-mm diamond bit, the lateral portions of the lamina and medial facet joint are drilled, under constant irrigation, to eggshell thickness. The remaining cortical bone and ligamentum flavum are removed using a curette or Kerrison punch to reveal the underlying fibroareolar layer. The epidural venous plexus is coagulated using bipolar cautery, beginning medially and extending along the nerve root sleeve. It is then divided sharply to expose the thecal sac and exiting nerve root. Soft-disc herniations are usually located in the axilla of the nerve root. If excessive nerve root manipulation is necessary or the foraminal volume limited, pediculotomy is performed. With the nerve root protected and under direct vision, the superomedial aspect of the inferior pedicle is resected using the same 3-mm diamond bit (Fig. 1). The annulus is then incised using a No. 15 blade, and the compressive disc fragments and osteophytes are removed using pituitary forceps or curettage without excessive retraction of the nerve root (Fig. 2). Hemostasis is achieved and the wound is closed.

\section{SUMMARY}

The combined posterior cervical hemilaminectomy, foraminotomy, and discectomy is a well-established and effective surgical procedure for the treatment of cervical radiculopathy. The addition of a limited microsurgical pediculotomy to the standard posterior approach is a useful adjunct that allows for easier and more complete excision of the compressive elements without requiring exces- 


\section{Adjunctive pediculotomy}

sive traction on the nerve root and epidural venous plexus while providing a more generous decompression of the neural foramen in cases in which discectomy is not performed.

\section{References}

1. Aldrich F: Posterolateral microdiscectomy for cervical monoradiculopathy caused by posterolateral soft cervical disc sequestration. J Neurosurg 72:370-377, 1990

2. Epstein JA, Lavine LS, Aronson HA, et al: Cervical spondylotic radiculopathy: the syndrome of foraminal constriction treated by foramenotomy and the removal of osteophytes. Clin Orthop 40:113-122, 1965

3. Gore DR, Sepic SB: Anterior discectomy and fusion for painful cervical disc disease. A report of 50 patients with an average follow-up of 21 years. Spine 23:2047-2051, 1998

4. Henderson CM, Hennessy RG, Shuey HM Jr, et al: Posteriorlateral foraminotomy as an exclusive operative technique for cervical radiculopathy: a review of 846 consecutively operated cases. Neurosurgery 13:504-512, 1983
5. Hilibrand AS, Carlson GD, Palumbo MA, et al: Radiculopathy and myelopathy at segments adjacent to the site of a previous anterior cervical arthrodesis. J Bone Joint Surg Am 81: 519-528, 1999

6. Krupp W, Schattke H, Muke R: Clinical results of the foraminotomy as described by Frykholm for the treatment of lateral cervical disc herniation. Acta Neurochir 107:22-29, 1990

7. Kumar GR, Maurice-Williams RS, Bradford R: Cervical foraminotomy: an effective treatment for cervical spondylotic radiculopathy. Br J Neurosurg 12:563-568, 1998

8. Williams RW: Microcervical foraminotomy. A surgical alternative to intractable radicular pain. Spine 8:708-716, 1983

Manuscript received November 20, 2001.

Accepted in final form December 4, 2001.

Address reprint requests to: K. Michael Webb, M.D., Department of Neurosurgery, University of Virginia, HSC Box 212, Charlottesville, Virginia 22908-0212. email: kw3a@ hscmail.mcc.virginia.edu. 